\title{
Tangent bundle embeddings of manifolds in Euclidean space
}

\author{
Mohammad Ghomi*
}

\begin{abstract}
For a given $n$-dimensional manifold $M^{n}$ we study the problem of finding the smallest integer $N\left(M^{n}\right)$ such that $M^{n}$ admits a smooth embedding in the Euclidean space $\mathbb{R}^{N}$ without intersecting tangent spaces. We use the Poincaré-Hopf index theorem to prove that $N\left(\mathbb{S}^{1}\right)=4$, and construct explicit examples to show that $N\left(\mathbb{S}^{n}\right) \leq 3 n+3$, where $\mathbb{S}^{n}$ denotes the $n$-sphere. Finally, for any closed manifold $M^{n}$, we show that $2 n+1 \leq N\left(M^{n}\right) \leq 4 n+1$.
\end{abstract}

Mathematics Subject Classification (2000). 53A07, 57R40.

Keywords. Submanifold, embedding, tangent bundle, tangent developable, skew loop, totally skew submanifold, T-embedding.

\section{Introduction}

Every $C^{1}$-immersion $f: M^{n} \rightarrow \mathbb{R}^{N}$, where $M^{n}$ is an $n$-manifold and $\mathbb{R}^{N}$ is the Euclidean $N$-space, induces a mapping of the tangent bundle $T M$ via the differential map $d f: T M \rightarrow \mathbb{R}^{N}$. We say that $f$ is a tangent bundle embedding, or a T-embedding for short, provided that $d f$ is one-to-one. In other words, a submanifold of Euclidean space is T-embedded provided that it has no pairs of intersecting tangent spaces. The aim of this note is to begin the study of and call attention to the following basic question:

Problem 1.1. For a given manifold $M^{n}$, what is the smallest integer $N\left(M^{n}\right)$ such that $M^{n}$ admits a T-embedding in $\mathbb{R}^{N}$ ?

The above problem may be regarded as a generalization of the investigations conducted in the 1940's by H. Whitney [12], culminating in his celebrated theorem that every $n$-manifold may be embedded in $\mathbb{R}^{2 n}$. The prime stimulus for this work, however, is due to the recent renewed interest in studying global geometry of tangent

\footnotetext{
*The research of the author was supported in part by NSF Grant DMS-0204190 and CAREER award DMS0332333
} 
lines of closed curves and knots ([1], [2], [4], [11], [13]), see Note 1.8. Our first result shows that the circle $\mathbb{S}^{1}$ admits no T-embedding in $\mathbb{R}^{3}$.

Theorem 1.2. Every closed $C^{1}$-immersed curve in $\mathbb{R}^{3}$ has uncountably many pairs of intersecting tangent lines.

The proof of Theorem 1.2, which we present in Section 2, follows from the Poincaré-Hopf index theorem (Proposition 2.3) together with a perturbation argument for bitangent planes (Proposition 2.4). The remaining results of this paper, which are proved in Section 3, are concerned mainly with some explicit constructions of Tembeddings:

Theorem 1.3. Every torus $\mathbb{T}^{n}$ admits a smooth T-embedding in $\mathbb{R}^{4 n}$ given by

$$
\mathbb{C}^{n} \supset \mathbb{T}^{n} \ni\left(z_{1}, \ldots, z_{n}\right) \longmapsto\left(z_{1}, z_{1}^{2}, \ldots, z_{n}, z_{n}^{2}\right) \in \mathbb{C}^{2 n} .
$$

In particular, there exists a T-embedded closed curve in $\mathbb{R}^{4}$.

Where $\mathbb{C}^{n}$ denotes the complex $n$-space. The previous two theorems solve Problem 1.1 for the case $M^{n}=\mathbb{S}^{1}$ :

Corollary 1.4. $N\left(\mathbb{S}^{1}\right)=4$.

Another class of T-embeddings may be constructed using cubic curves, and exploiting the fact (Proposition 3.2) that T-embeddings are preserved under cartesian product:

Theorem 1.5. Let $f: M^{n} \rightarrow \mathbb{R}^{N}$ be any $C^{1}$-embedding, and $f_{i}, i=1, \ldots, N$ be the components of $f$. Then

$$
M \ni p \longmapsto\left(f_{1}(p), f_{1}^{2}(p), f_{1}^{3}(p), \ldots, f_{N}(p), f_{N}^{2}(p), f_{N}^{3}(p)\right) \in \mathbb{R}^{3 N}
$$

is a T-embedding. In particular, $N\left(\mathbb{R}^{n}\right) \leq 3 n$ and $N\left(\mathbb{S}^{n}\right) \leq 3 n+3$.

Since every planar curve has intersecting tangents, the above theorem immediately yields that $N\left(\mathbb{R}^{1}\right)=3$. Further, note that the above result, via Whitney's $2 n$-embedding theorem [12], implies that any $n$-manifold admits a T-embedding in $\mathbb{R}^{6 n}$. Working a bit harder, via successive projections into subspaces of lower dimension, we obtain:

Corollary 1.6. $N\left(M^{n}\right) \leq 4 n+1$, for any manifold $M^{n}$. 
Using Thom's transversality theorem, it can be shown that the above result is in fact a generic property [5]. That is, any immersion of $M^{n}$ in $\mathbb{R}^{4 n+1}$ can be turned into a T-embedding by an arbitrarily small perturbation. In Section 3, we also obtain a lower bound:

Theorem 1.7. $N\left(M^{n}\right) \geq 2 n+1$, for any non-contractible manifold $M^{n}$.

Note that since $\operatorname{dim}\left(T M^{n}\right)=2 n$, we trivially have $N\left(M^{n}\right) \geq 2 n$ for all manifolds. The last theorem improves this lower bound for manifolds whose homotopy type is different from that of a point. In particular, when $M^{n}$ is a compact manifold without boundary, $N\left(M^{n}\right) \geq 2 n+1$. However, the author does not know if the noncontractibility assumption in Theorem 1.7 is necessary. More generally, the author does not know if the estimates in the last two results can be improved. But it would be reasonable to conjecture that $N\left(M^{n}\right) \leq 4 n$.

Note 1.8 (Terminology and some history). The class of mappings we study in this paper, the T-embeddings, are not to be confused with skew immersions, or S-immersions ([1], [2], [4], [11], [13], [8]), which are defined as immersions without any pairs of parallel tangent lines. The first proof of the existence of an S-embedding of a circle, or skew loop, in $\mathbb{R}^{3}$ is due to B. Segre [8]. For an explicit formula for such a curve see [2]; there skew loops where used to solve Wente's shadow problem which is related to stability questions concerning surfaces of constant mean curvature [3]. Skew loops are also of interest due to their connection with quadric surfaces: the author and B. Solomon [4] showed that the absence of skew loops characterizes ellipsoids, and S. Tabachnikov [11] has ruled out the existence of skew loops on any quadric surface.

One may also introduce a notion of totally skew embedding, or TS-embedding [5], which is defined as an embedding which is both a T-embedding and an S-embedding; an example is the cubic curve $x \longmapsto\left(x, x^{2}, x^{3}\right)$, and another example is given by Proposition 3.1. Though in this paper we confine our attention primarily to Tembeddings, Problem 1.1 can be stated for S-embeddings and TS-embeddings as well. The case of TS-embeddings will be studied in [5], and is related to the existence of nonsingular bilinear maps, and the "generalized vector field problem". The present work has a different flavor which is in part due to the fact that, unlike T-embeddings, TS-embeddings are not preserved under cartesian product (see Note 3.3).

\section{Proof of Theorem 1.2}

The basic idea for the proof of Theorem 1.2 is as follows. Let $C \subset \mathbb{R}^{3}$ be (the image of) a closed $C^{1}$-immersed curve. For every unit vector $u \in \mathbb{S}^{2}$, let $\Pi(u, \lambda)$ be the 
plane which is orthogonal to $u$ and passes through $\lambda u$; that is,

$$
\Pi(u, \lambda):=\left\{x \in \mathbb{R}^{3}:\langle x-\lambda u, u\rangle=0\right\},
$$

where $\langle\cdot, \cdot\rangle$ denotes the standard inner product. Let $\lambda_{u}$ be the infimum of all $\lambda$ such that $\Pi(u, \lambda)$ is disjoint from $C$. Then $\Pi_{u}:=\Pi\left(u, \lambda_{u}\right)$ is tangent to $C$. Let $L_{u}$ be the collection of all tangent lines of $C$ which lie in $\Pi_{u}$. We claim that, for some $u \in \mathbb{S}^{2}, L_{u}$ must contain a pair of intersecting lines. Suppose not. Then, for each $u \in \mathbb{S}^{2}$, let $\ell_{u}$ be a line which passes through $u$ and is parallel to the elements of $L_{u}$. Since $\Pi_{u}$ is orthogonal to $u$, this yields a tangent line field on $\mathbb{S}^{2}$. As we show in Lemma 2.1 below, $u \mapsto \ell_{u}$ is continuous. But it follows from the Poincaré-Hopf index theorem (Lemma 2.2), that there are no continuous line fields on the sphere. Hence we obtain a contradiction. So at least one of the planes $\Pi_{u}$ must have contained a pair of intersecting tangent lines. A perturbation argument (Proposition 2.3) applied to $\Pi_{u}$ then yields the existence of infinitely many pairs of intersecting tangent lines in nearby bitangent planes, and completes the proof.

To proceed more formally, let $c: \mathbb{S}^{1} \simeq \mathbb{R} / 2 \pi \mathbb{Z} \rightarrow \mathbb{R}^{3}$ be a $C^{1}$ unit speed curve, i.e., a $C^{1}$ mapping with $\left\|c^{\prime}\right\|=1$. For any $u \in \mathbb{S}^{2}$, let $t_{u} \in \mathbb{S}^{1}$ be a maximum point of $t \mapsto\langle c(t), u\rangle$, and set $\ell(u):=\left\{ \pm c^{\prime}\left(t_{u}\right)\right\}$.

Lemma 2.1. Suppose that $c^{\prime}\left(t_{1}\right)= \pm c^{\prime}\left(t_{2}\right)$ whenever $t_{1}$ and $t_{2}$ are maximum points of $t \mapsto\langle c(t), u\rangle$. Then $\ell: \mathbb{S}^{2} \rightarrow \mathbb{S}^{2} /\{ \pm 1\} \simeq \mathbb{R} \mathbb{P}^{2}$ is well-defined and continuous.

Proof. Our hypothesis, together with the definition of $\ell$, implies that

$$
\ell(u)=\left\{ \pm c^{\prime}\left(t_{u}\right)\right\} \Longleftrightarrow\langle c(t), u\rangle \leq\left\langle c\left(t_{u}\right), u\right\rangle, \text { for all } t \in \mathbb{S}^{1} .
$$

Thus $\ell$ is well-defined. To prove the continuity of $\ell$, let $u_{i} \in \mathbb{S}^{2}$ be a sequence, and $t_{u_{i}}$ be a maximum point of $t \mapsto\left\langle c(t), u_{i}\right\rangle$. Then

$$
\ell\left(u_{i}\right)=\left\{ \pm c^{\prime}\left(t_{u_{i}}\right)\right\}
$$

Since $\mathbb{S}^{1}$ is compact, $t_{u_{i}}$ has a limit point $\bar{t}$. Thus, since $c$ is $C^{1}, c^{\prime}\left(t_{u_{i}}\right)$ has a limit point at $c^{\prime}(\bar{t})$. So, (2) yields that

$$
\left\{ \pm c^{\prime}(\bar{t})\right\} \text { is a limit point of } \ell\left(u_{i}\right) .
$$

Next note that (1) and (2) imply

$$
\left\langle c(t), u_{i}\right\rangle \leq\left\langle c\left(t_{u_{i}}\right), u_{i}\right\rangle .
$$

Now let $\bar{u}$ be a limit point of $u_{i}$. Then, since $\langle\cdot, \cdot\rangle$ is continuous, (4) implies

$$
\langle c(t), \bar{u}\rangle \leq\langle c(\bar{t}), \bar{u}\rangle .
$$


Consequently, (1) yields

$$
\ell(\bar{u})=\left\{ \pm c^{\prime}(\bar{t})\right\} .
$$

So, by (3), $\ell(\bar{u})$ is a limit point of $\ell\left(u_{i}\right)$; therefore, we conclude that $\ell$ is continuous.

We also need to recall the following well-known fact:

Lemma 2.2. There exists no continuous tangent line fields on $\mathbb{S}^{2}$.

Proof. By a generalization of the Poincaré-Hopf theorem on vector fields [10], the sum of the index of the singularities of a line field on a 2-manifold $M$ is equal to the Euler characteristic $\chi(M)$. In particular, if $M$ has a line field which is defined everywhere, i.e., it has no singularities, then we must have $\chi(M)=0$, which rules out $\mathbb{S}^{2}$.

Combining Lemmas 2.1 and 2.2, we obtain:.

Proposition 2.3. For every $C^{1}$ immersed curve $c: \mathbb{S}^{1} \rightarrow \mathbb{R}^{3}$, there exists a plane $\Pi \subset \mathbb{R}^{3}$ such that

(1) $c\left(\mathbb{S}^{1}\right)$ lies entirely on one side of $\Pi$

(2) c has a pair of tangent lines in $\Pi$ which intersect transversely

In particular, there exist $t_{0}, s_{0} \in \mathbb{S}^{1}$, such that $c\left(t_{0}\right), c\left(s_{0}\right) \in \Pi$ and $c^{\prime}\left(t_{0}\right) \times c^{\prime}\left(s_{0}\right) \neq 0$.

Proof. After a reparametrization, we may assume that $c$ has unit speed. For each $u \in \mathbb{S}^{2}$, let $t_{u}$ be a maximum point of $t \mapsto\langle c(t), u\rangle$, and let $\Pi_{u}$ be the plane given by

$$
\Pi_{u}:=\left\{x \in \mathbb{R}^{3}:\langle x, u\rangle=\left\langle c\left(t_{u}\right), u\right\rangle\right\} .
$$

Note that $t_{u}$ is a maximum point of $t \mapsto\langle c(t), u\rangle$ if and only if $c\left(t_{u}\right) \in \Pi_{u}$.

Suppose now that $c$ has no pairs of intersecting tangent lines in $\Pi_{u}$. Then at all the maximum points $t_{u}$ of $t \mapsto\langle c(t), u\rangle$, the tangent vectors $c^{\prime}\left(t_{u}\right)$ should be equal up to a sign. So, by Lemma 2.1 , the mapping $\ell: \mathbb{S}^{2} \rightarrow \mathbb{S}^{2} /\{ \pm 1\}$, given by $\ell(u):=\left\{ \pm c^{\prime}\left(t_{u}\right)\right\}$, is well defined and continuous. But $\left\langle c^{\prime}\left(t_{u}\right), u\right\rangle=0$, so $\ell$ determines a tangent line field on $\mathbb{S}^{2}$, given by

$$
L_{u}:=\{u+\lambda v: v \in \ell(u), \text { and } \lambda \in \mathbb{R}\} .
$$

This contradicts Lemma 2.2 and thus completes the proof.

Next we show that it is possible to perturb the plane $\Pi$ in the previous proposition to produce infinitely many pairs of intersecting tangent lines. For every $t \in \mathbb{S}^{1}$ and $\epsilon \in \mathbb{R}$ we define

$$
U_{\epsilon}(t):=\left\{x \in \mathbb{S}^{1}: \operatorname{dist}_{\mathbb{S}^{1}}(x, t)<\epsilon\right\},
$$


where dist $\mathbb{S}^{1}$ is the intrinsic distance in $\mathbb{S}^{1}$. The end points of the above interval are denoted by $\partial U_{\epsilon}(t)$.

Proposition 2.4. Let $c: U_{\eta}\left(t_{0}\right) \cup U_{\delta}\left(s_{0}\right) \rightarrow \mathbb{R}^{3}$ be a $C^{1}$ immersion for some $t_{0}$, $s_{0} \in \mathbb{S}^{1}, t_{0} \neq s_{0}$, and $\eta, \delta>0$. Suppose that there exists a plane $\Pi \subset \mathbb{R}^{3}$ such that

(1) $c\left(U_{\eta}\left(t_{0}\right) \cup U_{\delta}\left(s_{0}\right)\right)$ lies on one side of $\Pi$;

(2) $c\left(t_{0}\right), c\left(s_{0}\right) \in \Pi$;

(3) $c^{\prime}\left(t_{0}\right) \times c^{\prime}\left(s_{0}\right) \neq 0$;

(4) For every $0<\alpha \leq \delta$, there is a $0<\beta<\alpha$ such that $c\left(\partial U_{\beta}\left(s_{0}\right)\right) \cap \Pi=\emptyset$.

Then there exists an $0<\epsilon \leq \eta$ with the following property: For every $t \in U_{\epsilon}\left(t_{0}\right)$ there exists an $s \in U_{\delta}\left(s_{0}\right)$ such that the tangent lines of $c$ at $c(t)$ and $c(s)$ intersect transversely.

Proof. Since $(t, s) \mapsto c^{\prime}(t) \times c^{\prime}(s)$ is continuous and $c^{\prime}\left(t_{0}\right) \times c^{\prime}\left(s_{0}\right) \neq 0$, we may choose $\epsilon$ small enough so that

$$
c^{\prime}(t) \times c^{\prime}(s) \neq 0, \quad \text { for all }(t, s) \in U_{\epsilon}\left(t_{0}\right) \times U_{\epsilon}\left(s_{0}\right) .
$$

In particular, $U_{\epsilon}\left(t_{0}\right) \cap U_{\epsilon}\left(s_{0}\right)=\emptyset$. Further, item 4 above implies that we may assume

$$
c\left(\partial U_{\epsilon}\left(s_{0}\right)\right) \cap \Pi=\emptyset,
$$

where $\partial U$ denotes the endpoints of $U$.

Now let $\ell_{x}$ denote the tangent line of $c$ at $c(x)$. Using (6), and the assumption that $c^{\prime}\left(t_{0}\right) \times c^{\prime}\left(s_{0}\right) \neq 0$, we may rotate $\Pi$ around $\ell_{t_{0}}$ to obtain a new tangent plane, say $\Pi_{t_{0}}$, such that

$$
\Pi_{t_{0}} \text { separates } c\left(s_{0}\right) \text { from } c\left(\partial U_{\epsilon}\left(s_{0}\right)\right) \text {. }
$$

That is, $c\left(s_{0}\right)$ lies in one of the open half-spaces determined by $\Pi_{t_{0}}$ while $c\left(\partial U_{\epsilon}\left(s_{0}\right)\right)$ lies in the other open half-space.

Let $n_{0}$ denote a unit normal to $\Pi_{t_{0}}$, and $n: U_{\epsilon}\left(t_{0}\right) \rightarrow \mathbb{S}^{2}$ be any continuous unit normal vector field of $c$ with $n\left(t_{0}\right)=n_{0}$. For every $t \in U_{\epsilon}\left(t_{0}\right)$, let $\Pi_{t}$ be the plane which pass through $c(t)$ and is perpendicular to $n(t)$. Then, by continuity of $t \mapsto \Pi_{t}$, (7) implies that there exists $0<\epsilon^{\prime} \leq \epsilon$ such that

$$
\Pi_{t} \text { separates } c\left(s_{0}\right) \text { from } c\left(\partial U_{\epsilon}\left(s_{0}\right)\right),
$$

for all $t \in U_{\epsilon^{\prime}}\left(t_{0}\right)$.

So, for each $t \in U_{\epsilon^{\prime}}\left(t_{0}\right)$, we may rotate $\Pi_{t}$ around $\ell_{t}$ until we obtain a plane, say $\Pi_{t}^{\prime}$, which is tangent to $c\left(U_{\epsilon}\left(s_{0}\right)\right)$. Then, in addition to $\ell_{t}, \Pi_{t}^{\prime}$ will contain another tangent line $\ell_{s}$, for some $s \in U_{\epsilon}\left(s_{0}\right)$. Thus (5) implies that $\ell_{t}$ and $\ell_{s}$ intersect transversely, which completes the proof. 
The above propositions now yield:

Proof of Theorem 1.2. For any $C^{1}$ immersed closed curve $c: \mathbb{S}^{1} \rightarrow \mathbb{R}^{3}$, Proposition 2.3 ensures that all the conditions of Proposition 2.4 are satisfied except possibly Condition 4. If Condition 4 is also satisfied, then Proposition 2.4 yields that $c$ has uncountably many intersecting tangent lines. On the other hand, if Condition 4 is not satisfied, then it follows that $c$ maps an open neighborhood of $\mathbb{S}^{1}$ into $\Pi$. In particular, $c$ is tangent to $\Pi$ uncountably often. But this again results in uncountably many intersecting tangents, because $\Pi$ contains a pair of nonparallel tangent lines.

Note 2.5. It is comparatively easy to prove the existence of intersecting tangents for knotted curves $c: \mathbb{S}^{1} \rightarrow \mathbb{R}^{3}$. To see this let $d$ be the maximum distance of $c\left(\mathbb{S}^{1}\right)$ from the origin $o:=(0,0,0)$, and $S \subset \mathbb{R}^{3}$ be a sphere of radius $r>d$ centered at $o$. Then, for each $t \in \mathbb{S}^{1}$, the ray

$$
R_{t}:=\left\{c(t)+\lambda c^{\prime}(t): \lambda \geq 0\right\}
$$

has a point inside and a point outside of $S$. So $R_{t}$ intersects $S$ at a unique point, say $c(t)+\lambda_{t} c^{\prime}(t)$. Since $c$ is continuous, $t \mapsto \lambda_{t}$ is continuous as well. Thus

$$
\bar{c}:=c(t)+\lambda_{t} c^{\prime}(t)
$$

gives a closed curve in $S$. Suppose that $c$ has no pairs of intersecting tangents. Then

$$
R_{t} \cap R_{s}=\varnothing
$$

for all $t \neq s$. Consequently $\bar{c}$ is one-to-one. So, by Jordan's curve theorem, $\bar{c}\left(\mathbb{S}^{1}\right)$ bounds an embedded disk, and is therefore unknotted. Now define $h: \mathbb{S}^{1} \times[0,1] \rightarrow$ $\mathbb{R}^{3}$ by

$$
h(t, s):=c(t)+s \lambda_{t} c^{\prime}(t) .
$$

Clearly $h$ is continuous. Further, (8) implies that $h$ is one-to-one. Thus $h$ gives an isotopy between $c\left(\mathbb{S}^{1}\right)$ and $\bar{c}\left(\mathbb{S}^{1}\right)$, which is a contradiction. So we conclude that $c$ must have a pair of intersecting tangent lines

Note 2.6. The set of all tangent lines to a $C^{1}$ curve $c: \mathbb{S}^{1} \rightarrow \mathbb{R}^{3}$ determines a surface parametrized by $c(t)+s c^{\prime}(t)$, and called the tangent developable of $c$. Theorem 1.2 implies that the tangent developable of any closed $C^{1}$ curve in $\mathbb{R}^{3}$ has infinitely many double points. The multiple points and singularities of the tangent developable of "generic" curves have been studied by a number of authors. For a survey of results of this type and references see [7]. 


\section{Proof of other results and examples}

In the previous section we established the nonexistence of closed T-embedded curves in $\mathbb{R}^{3}$. The following result shows that such curves may be constructed in $\mathbb{R}^{4}$.

Proposition 3.1. The mapping $c: \mathbb{S}^{1} \rightarrow \mathbb{R}^{4}$, given by

$$
\mathbb{C} \supset \mathbb{S}^{1} \ni z \stackrel{c}{\longmapsto}\left(z, z^{2}\right) \in \mathbb{C}^{2},
$$

is a TS-embedding.

Proof. We may parametrize $c$ as

$$
c(t):=(\cos t, \sin t, \cos 2 t, \sin 2 t) .
$$

First we verify the skewness. To see this let

$$
T(t):=\frac{c^{\prime}(t)}{\left\|c^{\prime}(t)\right\|}=\frac{1}{\sqrt{3}}(-\sin t, \cos t,-2 \sin 2 t, 2 \cos 2 t) .
$$

It is enough to check that $T(t) \neq \pm T(s)$, unless $t \equiv s(\bmod 2 \pi)$. Suppose $T(t)=$ $T(s)$. Then $\sin t=\sin s$, and $\cos t=\cos s$. This yields $t \equiv s(\bmod 2 \pi)$. Next suppose that $T(t)=-T(s)$. Then $\sin t=-\sin s$, and $\cos t=-\cos s$. This yields

$$
t \equiv s+\pi(\bmod 2 \pi)
$$

But we also have $\sin 2 t=-\sin 2 s$, and $\cos 2 t=-\cos 2 s$. So

$$
2 t \equiv 2 s+\pi(\bmod 2 \pi) \text {. }
$$

Subtracting (9) from (10) we get $t \equiv s(\bmod 2 \pi)$. So $c$ is skew.

To see that $c$ is totally skew, note that

$$
c(t)=\left[\begin{array}{cccc}
\cos t & -\sin t & 0 & 0 \\
\sin t & \cos t & 0 & 0 \\
0 & 0 & \cos 2 t & -\sin 2 t \\
0 & 0 & \sin 2 t & \cos 2 t
\end{array}\right]\left[\begin{array}{l}
1 \\
0 \\
1 \\
0
\end{array}\right] .
$$

That is, $c$ is the image of a one parameter subgroup of the special orthogonal group $\mathrm{SO}(4)$ acting on $c(0)$. In particular, $c$ is invariant under such rotations. Thus to show that the tangent lines of $c$ do not intersect, it is enough to check that the tangent line of $c$ at $c(0)$ does not intersect any other tangent line. Let

$$
\begin{aligned}
\ell_{t}(s) & :=c(t)+s c^{\prime}(t) \\
& =(\cos t-s \sin t, \sin t+s \cos t, \cos 2 t-2 s \sin 2 t, \sin 2 t+2 s \cos 2 t) .
\end{aligned}
$$


be a parametrization for the tangent line of $c$ at $c(t)$. Setting $\ell_{t}\left(s_{1}\right)=\ell_{0}\left(s_{2}\right)$ yields:

$$
\left.\begin{array}{rl}
\cos t-s_{1} \sin t & =1, \\
\sin t+s_{1} \cos t & =s_{2}, \\
\cos 2 t-2 s_{1} \sin 2 t & =1, \\
\sin 2 t+2 s_{1} \cos 2 t & =2 s_{2} .
\end{array}\right\}
$$

Eliminating $s_{1}$ from the first and third equations, we get

$$
2 \sin 2 t \cos t-\sin t \cos 2 t=2 \sin 2 t-\sin t .
$$

Using the identities $\sin 2 t=2 \sin t \cos t$ and $\cos 2 t=1-2 \sin ^{2} t$, we may rewrite the above equation as

$$
2 \sin t(1-\cos t)^{2}=0 .
$$

The solutions to this equation, modulo $2 \pi$, are $t=0$ and $t=\pi$. But a quick examination of (11) reveals that only $t=0$ satisfies all the equations. Thus the tangent line $\ell_{0}$ is disjoint from all other tangent lines of $c$, and we conclude that $c$ is totally skew.

Proposition 3.2. If $M_{1} \subset \mathbb{R}^{n_{1}}$ and $M_{2} \subset \mathbb{R}^{n_{2}}$ are T-embedded submanifolds, then so is $M_{1} \times M_{2} \subset \mathbb{R}^{n_{1}+n_{2}}$.

Proof. Suppose, towards a contradiction, that $M_{1} \times M_{2}$ has intersecting tangent lines. Then there are distinct points $p, q$ in $M_{1} \times M_{2}$ and vectors $v \in T_{p}\left(M_{1} \times M_{2}\right)$ and $w \in T_{q}\left(M_{1} \times M_{2}\right)$, such that

$$
p+v=q+w .
$$

Note that $p=\left(p_{1}, p_{2}\right)$ and $q=\left(q_{1}, q_{2}\right)$ where $p_{i}, q_{i}$ are points in $M_{i}, i=1,2$. Further, $v=\left(v_{1}, v_{2}\right)$ and $w=\left(w_{1}, w_{2}\right)$ where $v_{i} \in T_{p_{i}} M_{i}$ and $w_{i} \in T_{q_{i}} M_{i}$. Thus the above equality implies that

$$
p_{1}+v_{1}=q_{1}+w_{1} \quad \text { and } \quad p_{2}+v_{2}=q_{2}+w_{2} .
$$

Since, by assumption, $p \neq q$, we must have $p_{1} \neq p_{2}$ or $q_{1} \neq q_{2}$. Thus the above equalities imply that $M_{1}$ or $M_{2}$ must have a pair of intersecting tangent lines respectively.

Note 3.3. In contrast to the previous proposition, $S$-embeddings are not closed under cartesian product. Indeed, the cartesian product of any two submanifolds will always have parallel tangent lines. To see this let $M_{1} \subset \mathbb{R}^{n_{1}}$ and $M_{2} \subset \mathbb{R}^{n_{2}}$ be a pair of submanifolds, and $p \in M_{1}, v \in T_{p} M_{1}$, and $q, r \in M_{2}$. Then $(v, 0) \in T_{(p, q)} M_{1} \times M_{2}$, and $(v, 0) \in T_{(p, r)} M_{1} \times M_{2}$. Thus $M_{1} \times M_{2}$ has parallel tangent vectors at all pairs of points $(p, r)$ and $(p, q)$. 
Combining the previous two propositions immediately yields Theorem 1.3. Further, the last proposition also yields:

Proof of Theorem 1.5. Any submanifold of a T-embedded submanifold is also Tembedded. Thus, by Proposition 3.2, it suffices to check that

$$
\mathbb{R} \ni x \mapsto\left(x, x^{2}, x^{3}\right) \in \mathbb{R}^{3}
$$

has no intersecting tangent lines. Suppose otherwise. Then there are $x, y \in \mathbb{R}, x \neq y$, such that

$$
\left(x, x^{2}, x^{3}\right)+\lambda\left(1,2 x, 3 x^{2}\right)=\left(y, y^{2}, y^{3}\right)+\mu\left(1,2 y, 3 y^{2}\right),
$$

for some $\lambda, \mu \in \mathbb{R}$. The above equality yields three equations:

$$
\begin{aligned}
x-y & =\mu-\lambda, \\
x^{2}-y^{2} & =2(\mu y-\lambda x), \\
x^{3}-y^{3} & =3\left(\mu y^{2}-\lambda x^{2}\right) .
\end{aligned}
$$

Solving for $y$ in the first equation and substituting in the second yields that $\lambda= \pm \mu$. If $\lambda=\mu$, then the first equation yields $x=y$, which is a contradiction. If $\lambda=-\mu$, then dividing the third equation by the first yields that

$$
x^{2}+x y+y^{2}=\frac{3}{2}\left(y^{2}+x^{2}\right)
$$

The above equation is equivalent to $(x-y)^{2}=0$, which yields $x=y$, another contradiction.

From this we immediately obtain the following result.

Corollary 3.4. If a manifold admits an embedding in $\mathbb{R}^{m}$, then it admits a T-embedding in $\mathbb{R}^{3 m}$.

Further, we can use Theorem 1.5 to etablish Corollary 1.6.

Proof of Corollary 1.6. As is well-known, every compact manifold $M^{n}$ admits a smooth embedding into $\mathbb{R}^{m}$, provided that $m$ is sufficiently large [6, p. 23]. Thus, by the previous corollary, $M^{n}$ admits a smooth embedding without intersecting tangents into $\mathbb{R}^{N}$, where $N=3 m$. If $N \leq 4 n+1$ we are done. So suppose that $N>4 n+1$. Define $g:(T M \times T M-\Delta T M) \rightarrow \mathbb{S}^{N-1}$, by

$$
g(x, y):=\frac{x-y}{\|x-y\|},
$$


where $\Delta$ denotes the diagonal elements of a cartesian product. Since

$$
\operatorname{dim}(T M \times T M-\Delta T M)=4 n<N-1=\operatorname{dim}\left(\mathbb{S}^{N-1}\right),
$$

and $g$ is a $C^{1}$ mapping, the image of $g$ must have measure zero [6, p. 68]. In particular, $g$ is not unto. Let $u \in \mathbb{S}^{N-1}$ be a vector in the complement of the image of $g, H_{u}$ be the hyperplane through the origin and orthogonal to $u$, and $\pi_{u}: \mathbb{R}^{N} \rightarrow H_{u}$ be the orthogonal projection

$$
\pi_{u}(x):=x-\langle x, u\rangle u .
$$

Then $\pi$ is an embedding on $T M$. But $\pi(T M)=T(\pi(M))$. Thus $\pi(M)$ has no intersecting tangent spaces, and we obtain a T-embedding of $M$ in $H_{u} \simeq \mathbb{R}^{N-1}$. We may repeat this procedure until we reach the desired dimension, $4 n+1$, for the ambient space.

Finally, we prove the last observation mentioned in the introduction. The proof below uses the notions of contractibility and retract of a topological space $X$. Recall that $X$ is contractible if there exists a point $x_{0} \in X$ and a continuous mapping $f: X \times[0,1] \rightarrow X$ such that $f(x, 0)=x$, and $f(x, 1)=x_{0}$. A subset $Y \subset X$ is called a retract of $X$ if there exists a continuous map $f: X \rightarrow Y$ such that $f(y)=y$ for all $y \in Y$.

Proof of Theorem 1.7. Suppose that there exists a non-contractible manifold $M^{n}$ which is T-embedded in $\mathbb{R}^{2 n}$. Since $\operatorname{dim}(T M)=2 n$, it follows from the theorem on the invariance of domain that $T M$ is an open subset of $\mathbb{R}^{2 n}$. We claim that $T M$ is closed in $\mathbb{R}^{2 n}$ as well. To see this let $p_{i}$ be a sequence of points in $T M$ converging to a point $p$ in $\mathbb{R}^{2 n}$. Let $q_{i}$ be the corresponding sequence of points in $M$ such that $p_{i} \in T_{q_{i}} M$. Since $M$ is compact, $q_{i}$ have a limit point $q$ in $M$. Since the tangent space $T_{q} M$ is a limit point of $T_{q_{i}} M$, it follows that $p \in T_{q} M \subset T M$. Thus $T M$ is both open and closed in $\mathbb{R}^{2 n}$, which yields that $T M=\mathbb{R}^{2 n}$. In particular $T M$ is contractible. But $M$ is a retract of $T M$, and the retract of a contractible space is contractible. So $M$ is contractible - a contradiction.

Acknowledgments. The author thanks Bruce Solomon, Ralph Howard, and Serge Tabachnikov for stimulating and informative discussions.

\section{References}

[1] C. Adams, J. Othmer, A. Stier, C. Lefever, S. Pahk, and J. Tripp, An introduction to the supercrossing index of knots and the crossing map. J. Knot Theory Ramifications 11 (2002), 445-459. Zbl 1003.57008 MR 1905697

[2] M. Ghomi, Shadows and convexity of surfaces. Ann. of Math. (2) 155 (2002), 281-293, 2002. Zbl 1013.53040 MR 1888801 
[3] M. Ghomi, Solution to the shadow problem in 3-space. In Minimal Surfaces, Geometric Analysis and Symplectic Geometry, Adv. Stud. Pure Math. 34, Math. Soc. Japan, Tokyo 2002, 129-142. Zbl 1031.53010 MR 1925735

[4] M. Ghomi and B. Solomon, Skew loops and quadric surfaces. Comment. Math. Helv. 77 (2002), 767-782. Zbl 1029.53002 MR 1949113

[5] M. Ghomi and S. Tabachnikov, Totally skew embeddings of manifolds in Euclidean space. Preprint.

[6] M. Hirsch, Differential Topology. Grad. Texts in Math. 33, Springer-Verlag, New York 1976. Zbl 0356.57001 MR 0448362

[7] G.-O. Ishikawa, Singularities of developable surfaces. In Singularity theory (Liverpool, 1996), London Math. Soc. Lecture Note Ser. 263, Cambridge University Press, Cambridge 1999, xxii-xxiii, 403-418. Zbl 0963.58017 MR 1709365

[8] B. Segre, Global differential properties of closed twisted curves. Rend. Sem. Mat. Fis. Milano 38 (1968), 256-263. Zbl 0186.55001 MR 0240754

[9] M. Spivak, A comprehensive introduction to differential geometry. Vol. I, second edition, Publish or Perish Inc., Wilmington, Del., 1979. Zbl 0439.53001 MR 0532830

[10] M. Spivak, A comprehensive introduction to differential geometry. Vol. III, second edition, Publish or Perish Inc., Wilmington, Del., 1979. Zbl 0439.53003 MR 0372756

[11] S. Tabachnikov, On skew loops, skew branes, and quadratic hypersurfaces. Moscow Math. J. 3 (2003), 681-690. Zbl 1050.53010 MR 2025279

[12] H. Whitney, The self-intersections of a smooth $n$-manifold in $2 n$-space. Ann. of Math. (2) 45 (1944), 220-246. Zbl 0063.08237 MR 0010274

[13] Y.-Q. Wu, Knots and links without parallel tangents. Bull. London Math. Soc. 34 (2002), 681-690. Zbl 1030.57013 MR 1924195

Received October 20, 2003; revised July 7, 2005

Mohammad Ghomi, School of Mathematics, Georgia Institute of Technology, Atlanta, GA 30332, U.S.A.

E-mail: ghomi@math.gatech.edu

URL: http://www.math.gatech.edu/ ghomi 